\title{
Characterization and Optimization of Isotachophoresis Parameters for Pacific Blue Succinimidyl Ester Dye on a PDMS Microfluidic Chip
}

\author{
Himali Somaweera ${ }^{1}$, Zachary Estlack ${ }^{2}{ }^{\mathbb{D}}$, Jasmine Pramila Devadhasan ${ }^{1}$, Jungtae Kim ${ }^{3}$ and \\ Jungkyu Kim 2,*(D) \\ 1 Department of Mechanical Engineering, Texas Tech University, Lubbock, TX 79409, USA; \\ himashcool@gmail.com (H.S.); jasminepramila.d@gmail.com (J.P.D.) \\ 2 Department of Mechanical Engineering, University of Utah, Salt Lake City, UT 84112, USA; \\ zachary.estlack@utah.edu \\ 3 KIST-EUROPE, 66123 Saarbrucken, Germany; tais@kist-europe.de \\ * Correspondence: jkim@mech.utah.edu; Tel.: +1-(801)-581-6743
}

Received: 16 September 2020; Accepted: 20 October 2020; Published: 22 October 2020

\begin{abstract}
Isotachophoresis (ITP) for Pacific Blue (PB) dye using a polydimethylsiloxane (PDMS) microfluidic chip is developed and characterized by determining the types and concentrations of electrolytes, the ITP duration, and the electric field density. Among candidate buffers for the trailing electrolyte (TE) and leading electrolyte (LE), $40 \mathrm{mM}$ borate buffer (pH 9) and $200 \mathrm{mM}$ trisaminomethane hydrochloride (Tris- $\mathrm{HCl})(\mathrm{pH} 8)$ were selected to obtain the maximum preconcentration and resolution of the PB bands, respectively. With the selected TE and LE buffers, further optimization was performed to determine the electric field (EF) density and the ITP duration. These ITP parameters showed a 20-170,000 preconcentration ratio from initial PB concentrations of $10 \mathrm{nM}-100 \mathrm{fM}$. Further demonstration was implemented to preconcentrate PB-conjugated lactate dehydrogenase (LDH) using the PDMS microfluidic chip. By utilizing the quenching nature of PB-LDH conjugation, we were able to identify concentrations of LDH as low as $10 \mathrm{ng} / \mathrm{mL}$. This simple PDMS microfluidic chip-based ITP for PB preconcentration enables highly sensitive biological and chemical analyses by coupling with various downstream detection systems.
\end{abstract}

Keywords: isotachophoresis; PDMS (polydimethylsiloxane) microfluidic chip; pacific blue succinimidyl ester; preconcentration; lactate dehydrogenase

\section{Introduction}

Analyzing low-abundance analytes in small volumes of biological samples is a primary challenge when performing highly sensitive biological and chemical analyses [1]. To overcome this challenge, efficient on-chip preconcentration and separation techniques such as field-amplified sample stacking (FASS) [2], dynamic pH junction [3], and isotachophoresis (ITP) [4] are widely used to improve the sensitivity and separation efficiency of downstream analytical tools. Recently, ITP utilizing a difference in electrophoretic mobilities has gained prominence as a means to achieve a million-fold preconcentration ratio by using fluorescence dyes [5-7].

To implement ITP with dye molecules properly in a microfluidic chip, a leading electrolyte (LE) and a trailing electrolyte (TE) should be selected along with electric field and duration [8]. A measurement point should also be selected to allow for accurate quantification of the preconcentration amount. Even though these ITP parameters for FITC and Alexa Fluor are well studied, each dye molecule should be characterized to optimize ITP preconcentration. Pacific Blue (PB) succinimidyl ester is an excellent fluorescent probe that is able to conjugate with primary amines by forming a stable carbodiimide 
bond [9]. The spectral and chemical properties of PB, such as its low pKa, high photostability, and high quantum yield, make PB a superior fluorescent dye and can provide a 200-fold increase in sensitivity with better resolution than fluorescamine derivatization $[10,11]$. In previous studies, PB has only been characterized for capillary zone electrophoresis (CZE) to detect amino acids with high sensitivity [10]. To preconcentrate PB using ITP, the selection of the TE and LE buffers with other ITP parameters are critical steps.

In addition, most microfluidic chips used for ITP are fabricated using glass and poly(methyl methacrylate) (PMMA) [12,13]. However, glass microfluidic chips require time-consuming and complicated wet-etching microfabrication processes that must be conducted in a cleanroom [14]. Furthermore, PMMA microfluidic chips require conventional prototyping, such as computer numerical controlled (CNC) milling [15,16], laser ablation [17], and micro-imprinting techniques [18]. In contrast, polydimethylsiloxane (PDMS) is commonly used to fabricate various microfluidic devices because of its low cost, ease of fabrication, biocompatibility, air permeability, and optical clarity [19,20]. Although there are some previous studies using PDMS microfluidic chips for ITP [21-25], they have not been widely adopted due to the hydrophobicity of PDMS surfaces and the unstable electroosmotic flow (EOF) due to non-crosslinked oligomers [20] though polyvinylpyrrolidone (PVP) has been used to mitigate this [24]. These issues must be resolved to achieve effective ITP preconcentrations in PDMS microfluidic chips.

In this study, by performing a parametric study, various ITP parameters such as the types and concentration of LE and TE buffers, electric field density, and duration were characterized to maximize the PB preconcentration ratio while maintaining spatial ITP resolution using a PDMS microfluidic chip. By analyzing the intensity and width of the PB bands, the optimum ITP conditions were determined to obtain preconcentration ratios from multiple initial PB concentrations in the PDMS microfluidic chip. Further demonstration was performed to preconcentrate and quantify PB-conjugated lactate dehydrogenase (LDH) using the optimized ITP conditions. This study provides a guideline to the experimental design and optimization of ITP to preconcentrate PB and PB-conjugated biomolecules with proper ITP resolution using a simple PDMS microfluidic chip that can easily be incorporated into a wider detection system.

\section{Materials and Methods}

\subsection{Fabrication and Preparation of PDMS Microfluidic Chip}

A PDMS microchannel (65 mm in length, $50 \mu \mathrm{m}$ width, and $25 \mu \mathrm{m}$ height) was designed and fabricated using a standard soft-lithography technique. First, with a photomask (CADART Inc., Bandon, OR., USA) from the microchannel design, molds were created on a 4-inch silicon wafer using SU-8 2015 (Kayaku Advanced Materials, Westborough, MA, USA) [26-28]. Uncured PDMS (Sylgard 184, Dow Corning, Midland, MI, USA) was then poured into the mold and cured at $95{ }^{\circ} \mathrm{C}$ for $1 \mathrm{~h}$ to replicate the microchannels. A featureless PDMS substrate was fabricated by pouring uncured PDMS into a petri dish and cured under the same conditions. After punching inlet and outlet holes, these two PDMS replicas were bonded using oxygen plasma activation. Finally, the PDMS microchannel device was bonded to a glass slide for structural support during the ITP.

The PDMS microchannel was then modified to improve the surface properties of the PDMS since the hydrophobicity and unstable EOF of PDMS are disadvantageous for ITP. The oxygen plasma activation, as well as an EOF suppressor, help minimize these drawbacks during ITP [20]. For this study, we chose polyvinylpyrrolidone (PVP), which is one of the water-soluble polymers commonly used as the EOF suppressor for ITP [24,29]. A 1\% (w/v) PVP (Sigma Aldrich, St. Louis, MO, USA) was prepared and added to all the electrolyte solutions for ITP experiments. 


\subsection{Reagent Preparation for ITP}

A TE stock solution of $200 \mathrm{mM}$ sodium tetraborate $\left(\mathrm{Na}_{2} \mathrm{~B}_{4} \mathrm{O}_{7}\right.$ : Borate) at $\mathrm{pH} 9$ was prepared by dissolving sodium tetraborate decahydrate and boric acid (Fisher Scientific, Pittsburgh, PA, USA) in DI water. Then, $5 \mathrm{mM}$ HEPES solution at $\mathrm{pH} 7$ was prepared using $1 \mathrm{M}$ HEPES (Invitrogen, Waltham, MA, USA). To select the LE, three LE stock solutions were initially prepared by titration: $1 \mathrm{M}$ sodium acetate ( $\mathrm{pH} 4$ ) (acetic acid and sodium acetate: Sigma Aldrich, St. Louis, MO, USA), 1 M sodium chloride (pH 7) (Sigma Aldrich, St. Louis, MO, USA), and $1 \mathrm{M}$ Tris-HCl (pH 9) (tris-base: Fisher Scientific, Pittsburgh, PA, USA, Hydrochloric acid: Macron Fine Chemicals, Center Valley, PA, USA). Tris-HCL was selected since it has been widely used for LE in ITP preconcentration. Other two LEs (sodium acetate and sodium chloride) were chosen as candidate LEs due to their high electrophoretic mobilities. The desired concentrations were achieved by diluting the stock solutions of the electrolytes and PB succinimidyl ester (Life Technologies, Rockville, MD, USA) to the appropriate levels. A $10 \mu \mathrm{M}$ stock was prepared using dimethyl sulfoxide (DMSO) (Sigma-Life Science, St. Louis, MO, USA). In addition, both a spacer and EOF suppressor should be selected to preconcentrate PB using ITP effectively. For this study, $100 \mathrm{nM}$ sodium acetate and PVP are used for a spacer and the suppressor for all ITP experiments and are mixed with the TE buffer.

\subsection{ITP Protocol}

First, a PDMS microchannel was filled with DI water and primed by applying an electric field $(77 \mathrm{~V} / \mathrm{cm})$ for $5 \mathrm{~min}$ to stabilize the surface charge on the PDMS surface. The separation channel was then washed three times with DI water and filled with the LE. Second, the sample inlet was rinsed with DI water 2-3 times and then filled with a PB sample prepared in the TE. As illustrated in Figure 1, during the ITP, a high-voltage electrode was inserted into the outlet (LE well), and a ground electrode was placed into the sample inlet (sample well). By using a custom-made high-voltage circuit (EMCO, Allen, TX, USA) with a DC amplifier (HY3003D, MASTECH, San Jose, CA, USA), various electric fields were applied to perform ITP. These procedures were used to determine the optimal electrolytes and electric field for preconcentrating PB using ITP. Between each ITP trial, the microchannel was washed with DI water. During ITP experiments, videos were collected at five different locations downstream from the sample inlet using an automated Ti-E fluorescence microscope (Nikon, Tokyo, Japan; Ti-E with an Andor iXon 3 CCD) and a 10× objective (Nikon, Tokyo, Japan; 0.3 NA) at a 200 ms exposure time. The frames were extracted from the video using a custom-made MATLAB 2019b program and analyzed using ImageJ 1.50 .

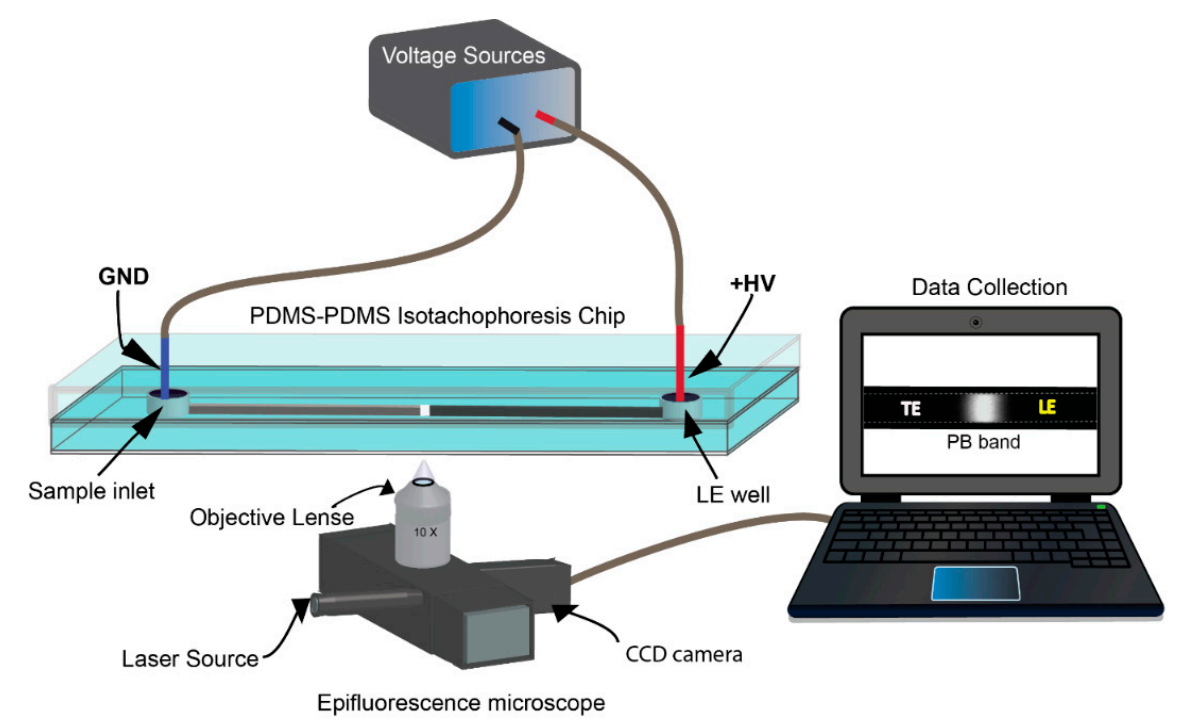

Figure 1. Schematic representation of the experimental setup in which Isotachophoresis (ITP) was 
used to preconcentrate Pacific blue succinimidyl ester dye (PB) using a simple PDMS microfluidic chip. ITP was performed using a computer-controlled voltage source after filling the sample inlet and outlet with the trailing electrolyte (TE) + PB and the leading electrolyte (LE) under an epi-fluorescence microscope.

\subsection{Determination of the TE and the LE Electrolytes}

To select a TE buffer, an initial trial of $5 \mathrm{mM} \mathrm{N}$-2-Hydroxyethylpiperazine-N'-2-Ethanesulfonic (HEPES) at pH 7 and $200 \mathrm{mM}$ borate buffer at pH 9 were used to evaluate ITP performance using $20 \mathrm{mM} \mathrm{NaCl}$ (pH 7) as a LE buffer. $\mathrm{NaCl}$ was chosen as the LE for the pilot study since it is known to have a high ionic mobility [30] and $123 \mathrm{~V} / \mathrm{cm}$ was used as the EF and measure the band intensity in the middle of the ITP channel. Once the proper TE type was selected that was able to stack PB effectively, different concentrations of TE were tested as initial trials (from 20 to $100 \mathrm{mM}$ ). Then, by using the selected TE, three possible LEs such as sodium acetate $(\mathrm{pH} 4)$, sodium chloride ( $\mathrm{pH} 7)$, and Tris- $\mathrm{HCl}$ ( $\mathrm{pH}$ ) of $100 \mathrm{mM}$ were evaluated to confirm the stacking effect of $\mathrm{Cl}^{-}$as a leading ion. The $\mathrm{pH}$ of all electrolytes was set near their $\mathrm{pKa}$ to have the best buffering capacity. After selection of the TE and LE types, the previously mentioned ITP protocol and setup (shown in Figure 1) was used to perform further parametric studies. All experiments in this text were performed in triplicate and all results have less than $5 \%$ error unless otherwise noted.

\subsection{Optimization of Electric Field (EF) and Measurement Point}

The focus of this study is to find the optimum EF that can provide a stable ITP environment without electrolysis. Since EF and measurement point are less sensitive to the concentration of LE and TE, once the proper LE and TE were selected, lower-end concentrations (100 mM LE and $40 \mathrm{mM}$ TE) were initially used for EF optimization. Using the ITP protocol mentioned in Section 2.3, the EF optimization was carried out using five different EF densities: 77, 92, 108, 123, and $138 \mathrm{~V} / \mathrm{cm}$ and measurements occurred at five different points in the channel. All experimental videos were recorded to obtain PB band profiles with respect to time to determine the overall ITP duration.

\subsection{Optimization of LE and TE Concentrations}

Once the optimum EF and measurement point were assigned, the optimum LE and TE concentrations were found. To evaluate the effect of LE and TE concentrations, first, five different concentrations of the selected LE buffer were tested: 100, 140, 175, 200, and $250 \mathrm{mM}$ with the selected TE buffer under an optimal EF as shown in SI. For each LE concentration, the fluorescence intensities, resolutions, separation efficiencies, and widths of the PB bands were measured at the $40 \mathrm{~mm}$ point and compared to determine the effect of LE concentration on ITP. The constant measurement location here was used because, regardless of buffer concentration, sample accumulation was poor prior to this point. Figure S1 shows that separation and resolution improve as the measurement location moves further from the sample inlet. In addition, it is seen that distance along the channel increases accumulation, but band length increases as well, ruling out the $50 \mathrm{~mm}$ point as well. After evaluating the effect of the LE concentration, three different concentrations of the selected TE $(40,60$, and $80 \mathrm{mM}$ borate buffer) were evaluated to investigate the effect of TE concentration on PB preconcentration under optimized EF, measurement point, and LE concentration.

\subsection{The Preconcentration Ratio of PB}

Once all ITP parameters were determined, a calibration curve (Figure S2) was constructed without ITP by measuring the intensities of known concentrations of PB 10, 25, 100, and 200 nM using a PDMS microfluidic chip. The separation channel was filled with the above concentrations of PB, and the fluorescence intensities were recorded for each concentration. To determine the preconcentration ratio, ITP was performed for five different initial concentrations of PB (10 nM, 1 nM, 100 pM, 10 pM, and $100 \mathrm{fM}$ ) under optimized ITP conditions. Linear regression of the calibration curve was used to 
calculate PB concentrations after ITP. All fluorescent images were background corrected and then analyzed using ImageJ.

\subsection{LDH Measurement with $P B$}

To demonstrate the preconcentration and separation of a PB-conjugated analyte using ITP, $200 \mu \mathrm{g} / \mathrm{mL}$ of L-LDH from rabbit muscle (Roche Diagnostics) was prepared in DI water. First, a series of $0.01,0.1,1$, and $100 \mu \mathrm{g} / \mathrm{mL} \mathrm{LDH}$ samples were prepared in the optimized TE buffer. Each LDH sample was then mixed with $0.34 \mu \mathrm{g} / \mathrm{mL}$ PB and incubated for $30 \mathrm{~min}$ for conjugation. Using the same ITP procedure mentioned above, ITP was then performed to demonstrate LDH preconcentration. In addition, to show the selectivity of this proposed method, preconcentration of LDH with PB was carried out using samples made in a mixture of 1:5 ratio of cell media to TE. The media was used in a HeLa dish culture and removed during conventional media changing. It consisted of Dulbecco's Modified Eagle Medium, Fetal Bovine Serum, and an antibiotic in a 100:10:1 ratio (Thermofisher Scientific, Waltham, MA, USA).

\section{Results and Discussion}

\subsection{Selecting Electrolytes to Preconcentrate PB on a PDMS Microfluidic Chip}

Using the PDMS microfluidic chip, we selected the proper TE and LE buffers to balance the electrophoretic mobility for $\mathrm{PB}$ preconcentration. $\mathrm{PB}$ samples were prepared in a TE buffer to obtain concentrated PB plugs in our anionic ITP. Additionally, due to the reactivity of PB with primary amines, the selection of a TE buffer must be non-amine [31]. As the electrophoretic mobility of PB is uncertain, a commonly used non-amine buffer N-2-Hydroxyethylpiperazine- $\mathrm{N}^{\prime}$-2-Ethanesulfonic (HEPES) $[5,32,33]$ was first evaluated using $\mathrm{Cl}^{-}\left(79 \times 10^{-9} \mathrm{~m}^{2} \mathrm{~V}^{-1} \mathrm{~s}^{-1}\right)$ as the leading ion. Although the electrophoretic mobility of HEPES at pH $7\left(0.7-1 \times 10^{-8} \mathrm{~m}^{2} \mathrm{~V}^{-1} \mathrm{~s}^{-1}\right)$ is significantly low compared to $\mathrm{Cl}^{-}$, HEPES was unable to provide the proper gradient of electrophoretic mobilities among TE, LE, and PB required to preconcentrate PB. This was unexpected due to the low mobility of HEPES, however parametric experiments rule out both $\mathrm{pH}$ and concentration effects. Further work is required to determine the true reason HEPES failed as a buffer for PB preconcentration in ITP. According to previous studies, borate was selected as the second candidate for the TE since borate buffers are commonly used for background electrolytes to analyze anions in capillary electrophoresis (CE), and borate has been used to detect amino acids coupled with PB using CE [10,34]. A borate buffer was tested, and a highly accumulated PB band was obtained. Although HEPES failed to preconcentrate PB experimentally, the electrophoretic mobility range of the borate ion $\left(30 \times 10^{-9} \mathrm{~m}^{2} \mathrm{~V}^{-1} \mathrm{~s}^{-1}\right)$ and the $\mathrm{Cl}^{-}$ ion was able to stack PB successfully $[31,33]$.

After selecting borate for the TE, we compared the fluorescence intensities of PB with three LE candidates $\left(\mathrm{NaCl}\right.$, Tris- $\mathrm{HCl}$, and $\left.\mathrm{NaCH}_{3} \mathrm{COO}^{-}\right)$to confirm the stacking effect with $\mathrm{Cl}^{-}$ion as the leading ion. Figure 2 presents the fluorescence intensity profiles of the $\mathrm{PB}$ band under the three different LE buffers. Out of the three different LEs, Tris- $\mathrm{HCl}$ showed the highest preconcentration of PB. Another aspect to select LE is to investigate peak resolution. Typically, PB shows two distinct peaks from hydrolysis from capillary electrophoresis [9]. As seen in Figure 2, two PB bands were formed with Tris- $\mathrm{HCl}$ at $\mathrm{pH} 8$ as the leading electrolyte along with borate as the trailing electrolyte. The preconcentration under tris-HCL was significant enough that the camera was saturated in the middle of the peak. The remaining ITP parameters for our chip design were optimized and the results are detailed in the supplemental information. 


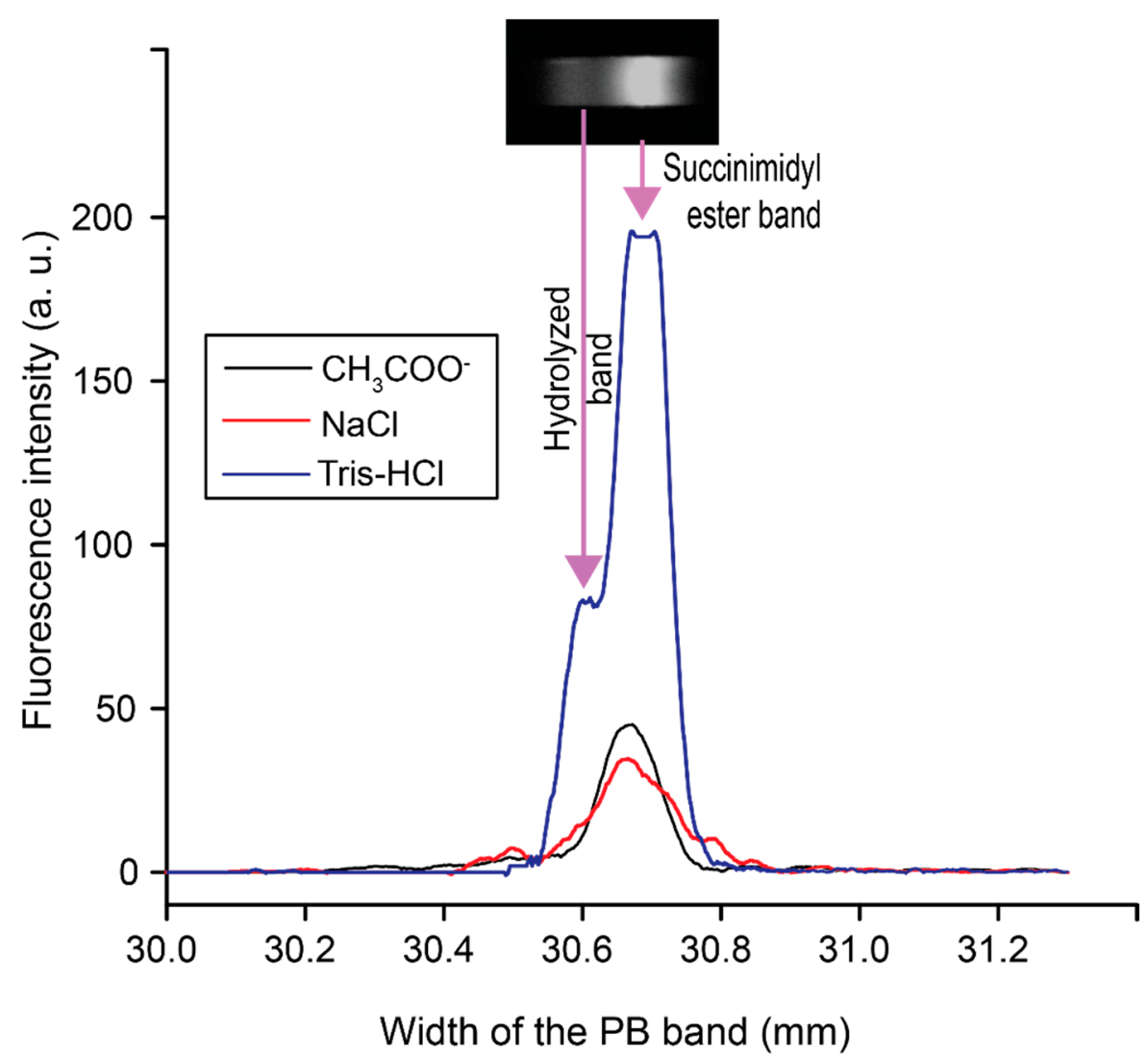

Figure 2. Selection of the leading electrolyte (LE) by evaluating the efficiencies of preconcentration and separation of $100 \mathrm{pM}$ PB. In total, $100 \mathrm{mM}$ of three different types of LE at $\mathrm{pH} 8$ (sodium acetate, sodium chloride, and Tris- $\mathrm{HCl}$ ) were used to preconcentrate $\mathrm{PB}$, while $40 \mathrm{mM}$ of borate buffer at $\mathrm{pH} 9$ was used as the TE under $123 \mathrm{~V} / \mathrm{cm}$. The intensity profiles of the PB band that formed at $30 \mathrm{~mm}$ downstream from the sample inlet is shown. The inset image illustrates the formation of two bands of PB due to hydrolysis.

\subsection{The Preconcentration Ratio of $P B$}

Using these optimal ITP parameters, the preconcentration ratio of PB was determined. Since the intensities of the PB bands were observed using a fluorescence microscope, a calibration curve (Figure S2) was obtained with a limit of detection of $9.2 \mathrm{nM}$ and this was utilized to quantify each concentrated PB band. Figure 3 shows the preconcentration ratios of $P B$ from various initial concentrations $\left(C_{i}\right)$ under ITP. Since ITP concentrates the low concentrations of analytes, the preconcentration ratio increases as the initial concentration decreases. The preconcentration ratios $\left(C_{i} / C_{f}\right)$ of $P B$ range from a factor of 20-170,000 times and were obtained from initial PB concentrations, based on dilution calculations, of $10 \mathrm{nM}$ to $100 \mathrm{fM}$ respectively. This corresponds to detected concentrations between 200 and $17 \mathrm{nM}$, showing that previously undetectable amounts of PB were concentrated to detectable levels in our system. In a previous study, Alexa Fluor showed a million-fold preconcentration ratio using ITP coupled with capillary electrophoresis in a glass microfluidic chip [35]. In this study, we utilized a simply fabricated PDMS straight channel as a separate channel to perform $10^{5}$-fold preconcentration of PB. 


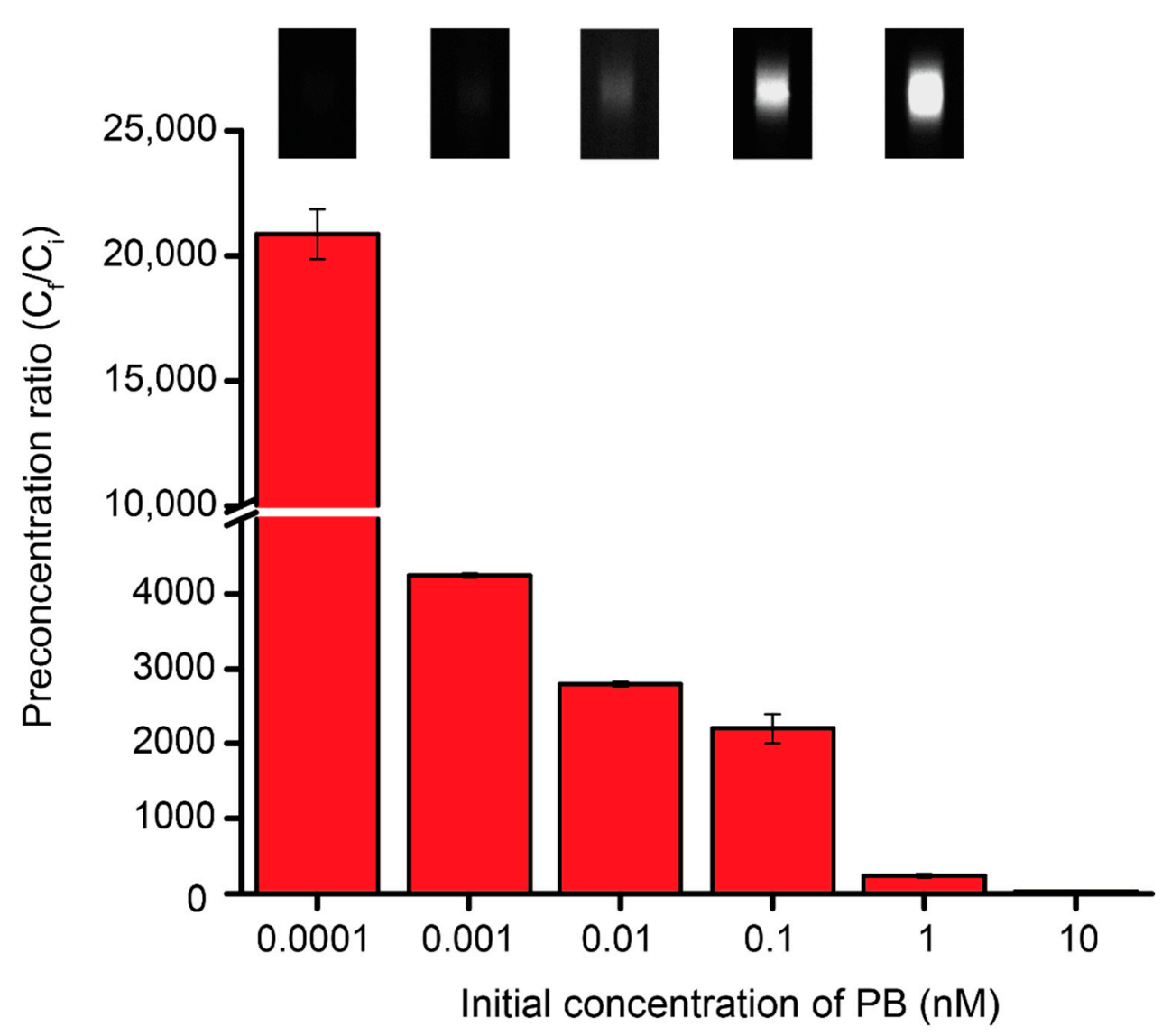

Figure 3. A plot of initial concentration of $\mathrm{PB}$ versus preconcentration ratio of different $\mathrm{PB}$ concentrations $(10,1,0.1,0.01,0.001$, and $0.0001 \mathrm{nM})$ prepared in $40 \mathrm{mM}$ borate buffer (TE). In total, $200 \mathrm{mM}$ Tris-HCl was used as the LE and $138 \mathrm{~V} / \mathrm{cm}$ as the EF. Inset images show the band formed during preconcentration.

\subsection{Preconcentrating LDH Using PB Conjugation}

To demonstrate the preconcentration of conjugated $\mathrm{PB}$ with a target analyte, we chose LDH to create the LDH-PB complex. LDH is a potential biomarker for different cancers [36] and many diseases, such as liver disease and myocardial infarction [37]. Although spectrometric methods have been reported to quantify LDH, LDH activity has often been measured by following either the oxidation of $\mathrm{NADH}$ with pyruvate or the reduction of $\mathrm{NAD}^{+}$, both of which involve several reaction steps $[38,39]$. In this work, by forming the PB-LDH complex with simple conjugation chemistry coupled with ITP, we successfully demonstrate how to quantify the amount of LDH using ITP. As shown in Figure 4A, the intensity of the fluorescent band decreased as the LDH concentration increased. By plotting the peak area of each LDH concentration, we obtained a calibration curve as a function of LDH concentrations, however camera saturation caused a plateau in the lowest concentration case. As seen in Figure 4B, the overall trend was an exponential decay with LDH concentration with a slight deviation at low concentrations due to the saturation. A previous study has shown the quenching effects that the protein itself can have on the fluorophore attached to it, using a series of Alexa Fluor dyes [40]. Therefore, the decrease of fluorescence intensity of PB may be due to the quenching effect of LDH that it has on PB; however, further study is needed. Using ITP under these optimized parameters, we were able to detect $\mathrm{LDH}$ concentrations in the range of $10 \mathrm{ng} / \mathrm{mL}$ to $100 \mu \mathrm{g} / \mathrm{mL}$. Furthermore, to show the selectivity of this purposed method, we also performed LDH measurement with used HeLa cell media as a background matrix. Even under these conditions, increasing LDH concentration decreased PB band intensity (Figure S3). Thus, our purposed method of indirect measurement of LDH can be used in real/authentic samples obtained from cell cultures. 
A

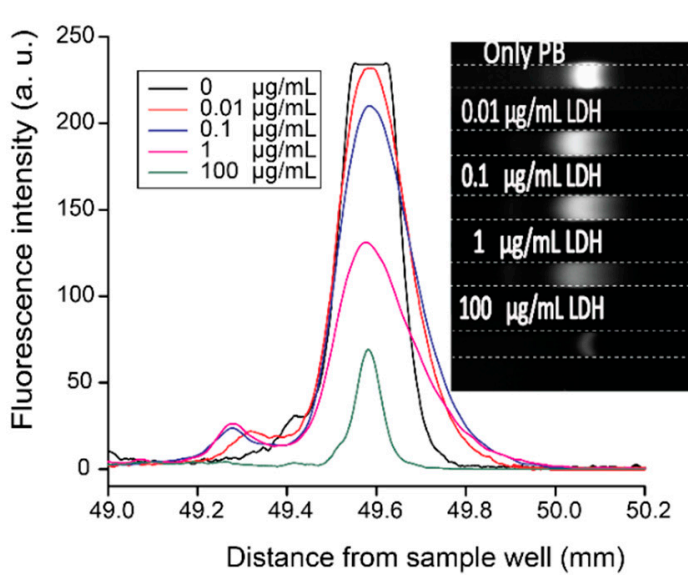

B

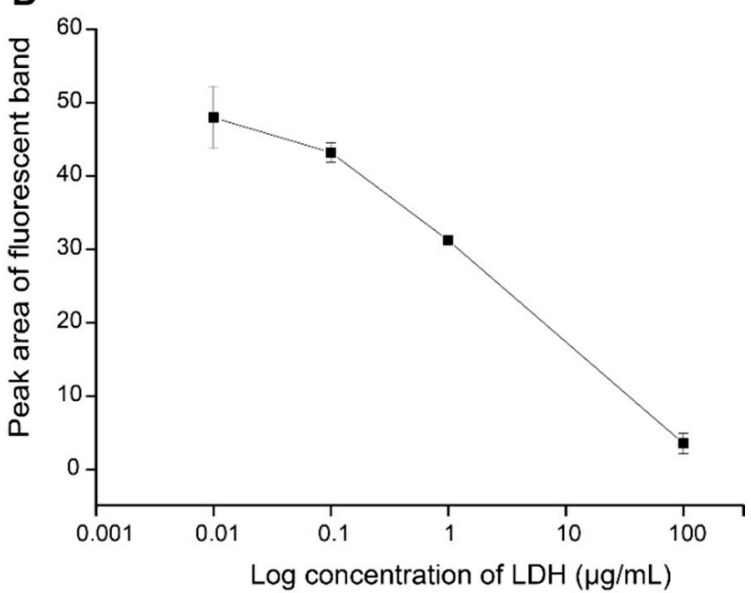

Figure 4. The indirect measurement of lactose dehydrogenase (LDH) using ITP. A series of LDH concentrations $(0.01,0.1,1$, and $100 \mu \mathrm{g} / \mathrm{mL})$ were prepared and conjugated with PB. In total, $200 \mathrm{mM}$ Tris- $\mathrm{HCl}$ for LE and $40 \mathrm{mM}$ borate buffer for TE were used under $138 \mathrm{~V} / \mathrm{cm}$. (A) The intensity profile of the PB band during the ITP process with various LDH concentrations. (B) The peak areas of the PB bands are plotted with the log of LDH concentration.

\section{Conclusions}

We performed an experimental parametric study focused on preconcentrating PB in a PDMS microfluidic chip by varying types of electrolyte, electric field density, measurement point, and electrolyte concentrations. An electrolyte system that was able to stack and separate PB was successfully characterized. The optimized parameters of the electrolyte system are as follows: $200 \mathrm{mM}$ Tris- $\mathrm{HCl}$ at $\mathrm{pH} 8$ as the LE, $40 \mathrm{mM}$ borate buffer at $\mathrm{pH} 9$ as the TE, $100 \mathrm{nM}$ sodium acetate as a spacer, and 1\% $(w / v)$ PVP as the electroosmotic flow suppressor. A spacer is required to ensure that the hydrolyzed PB band does not interfere with the normal PB band. As we used a PDMS device to perform ITP, our approach achieved lower cost, less complexity, and shorter device-fabrication time than commonly used glass microfluidic chips. Detecting a trace amount of PB was demonstrated with a maximum preconcentration ratio of $\approx 1.7 \times 10^{5}$ fold in $5 \mathrm{~min}$. In addition, we introduced a direct method to detect LDH concentrations ranging from $10 \mathrm{ng} / \mathrm{mL}$ to $100 \mu \mathrm{g} / \mathrm{mL}$ that differs from conventional colorimetric methods by using an indirect measurement of PB. The ITP system proposed here has significant potential as a low-cost, simple, and effective tool for detecting LDH.

Previous work on detecting amino acids using CE reported that amine conjugated PB increases the measurement sensitivity $[9,10,41,42]$. By using ITP preconcentration, we measured the PB signal as low as $100 \mathrm{fM}$ at given conditions of ITP. Coupling CZE with ITP can further enhance the measurement sensitivity of PB conjugated amines. In addition, both CZE and ITP require laborious sample preparation steps. Previously we demonstrated an automated CZE platform by using a programmable microfluidic platform (PMP) fabricated using soft lithography technique [27,43-45]. Since we characterized and optimized ITP conditions for PB in a PDMS microfluidic chip, this ITP system can be easily integrated with the PMP along with a laser-induced fluorescence (LIF) system $[26,42,43,46]$ to realize a fully automated chemical analyzer with extreme sensitivity. The integrated platform will be highly field-deployable for environmental monitoring, disease diagnostics, and exobiology studies.

Supplementary Materials: The following are available online at http://www.mdpi.com/2072-666X/11/11/951/s1, Figure S1: The impact of different EF densities on the accumulation of PB. LE and TE were $100 \mathrm{mM}$ Tris- $\mathrm{HCl}$ and $40 \mathrm{mM}$ borate buffer respectively, Table S1: Peak resolution at $30 \mathrm{~mm}$ and $40 \mathrm{~mm}$ measurement points under 138 $\mathrm{V} / \mathrm{cm} \mathrm{EF}$.

Author Contributions: Conceptualization, J.K. (Jungkyu Kim), H.S. and J.K. (Jungtae Kim); methodology, H.S., J.K. (Jungtae Kim), J.P.D., J.K. (Jungkyu Kim); software, H.S., Z.E.; validation, H.S., Z.E., J.K. (Jungtae Kim) and J.K. 
(Jungkyu Kim); formal analysis, H.S.; investigation, H.S., J.P.D., J.K. (Jungkyu Kim); resources, J.K. (Jungkyu Kim); writing-original draft preparation, H.S., J.K. (Jungkyu Kim); writing-review and editing, H.S., Z.E., J.K. (Jungtae Kim), J.K. (Jungkyu Kim); visualization, H.S., Z.E.; supervision, J.K. (Jungkyu Kim); project administration, J.K. (Jungkyu Kim); funding acquisition, J.K. (Jungkyu Kim). All authors have read and agreed to the published version of the manuscript.

Funding: This research was funded by KIST-Europe Joint Research Program and University of Utah. The APC was funded by the University of Utah.

Acknowledgments: Jungkyu Kim acknowledges the financial support of this project from University of Utah. Himali Somaweera and Jungtae Kim acknowledge the financial support of this project from the KIST-EUROPE Joint Research Grant. In addition, Jasmine Pramila Devadhasan and Jungkyu Kim are partially supported by Grant RP150720 from the Cancer Prevention Research Institute of Texas (CPRIT) and NASA NNX15AM98G. The authors thank Golden Kumar, N. Abidi, R. S. Dassanayake, and E. Rajakaruna at Texas Tech University for letting us use their facility.

Conflicts of Interest: The authors declare no conflict of interest.

\section{References}

1. Kleparnik, K.; Bocek, P. DNA diagnostics by capillary electrophoresis. Chem. Rev. 2007, 107, 5279-5317. [CrossRef] [PubMed]

2. Lichtenberg, J.; Verpoorte, E.; de Rooij, N.F. Sample preconcentration by field amplification stacking for microchip-based capillary electrophoresis. Electrophoresis 2001, 22, 258-271. [CrossRef]

3. Britz-McKibbin, P.; Chen, D.D.Y. Selective focusing of catecholamines and weakly acidic compounds by capillary electrophoresis using a dynamic pH junction. Anal. Chem. 2000, 72, 1242-1252. [CrossRef] [PubMed]

4. Walker, P.A., 3rd; Morris, M.D.; Burns, M.A.; Johnson, B.N. Isotachophoretic separations on a microchip. Normal Raman spectroscopy detection. Anal. Chem. 1998, 70, 3766-3769. [CrossRef] [PubMed]

5. Jung, B.; Bharadwaj, R.; Santiago, J.G. On-chip millionfold sample stacking using transient isotachophoresis. Anal. Chem. 2006, 78, 2319-2327. [CrossRef]

6. Liu, D.; Shi, M.; Huang, H.; Long, Z.; Zhou, X.; Qin, J.; Lin, B. Isotachophoresis preconcentration integrated microfluidic chip for highly sensitive genotyping of the hepatitis B virus. J. Chromatogr. B 2006, 844, 32-38. [CrossRef]

7. Eid, C.; Santiago, J.G. Isotachophoresis applied to biomolecular reactions. Lab Chip 2017, 18, 11-26. [CrossRef]

8. Bahga, S.S.; Santiago, J.G. Coupling isotachophoresis and capillary electrophoresis: A review and comparison of methods. Analyst 2013, 138, 735-754. [CrossRef]

9. Stockton, A.M.; Mora, M.F.; Cable, M.L.; Davenport, T.C.; Tilley, T.D.; Willis, P.A. Hydrolysis of 3-carboxy-6,8-difluoro-7-hydroxycoumarin (Pacific Blue (TM)) succinimidyl ester under acidic and basic conditions. Dyes Pigm. 2013, 96, 148-151. [CrossRef]

10. Chiesl, T.N.; Chu, W.K.; Stockton, A.M.; Amashukeli, X.; Grunthaner, F.; Mathies, R.A. Enhanced Amine and Amino Acid Analysis Using Pacific Blue and the Mars Organic Analyzer Microchip Capillary Electrophoresis System. Anal. Chem. 2009, 81, 2537-2544. [CrossRef]

11. Stockton, A.M.; Chiesl, T.N.; Lowenstein, T.K.; Amashukeli, X.; Grunthaner, F.; Mathies, R.A. Capillary Electrophoresis Analysis of Organic Amines and Amino Acids in Saline and Acidic Samples Using the Mars Organic Analyzer. Astrobiology 2009, 9, 823-831. [CrossRef] [PubMed]

12. Jacroux, T.; Bottenus, D.; Rieck, B.; Ivory, C.F.; Dong, W.J. Cationic isotachophoresis separation of the biomarker cardiac troponin I from a high-abundance contaminant, serum albumin. Electrophoresis 2014, 35, 2029-2038. [CrossRef] [PubMed]

13. Khurana, T.K.; Santiago, J.G. Preconcentration, separation, and indirect detection of nonfluorescent analytes using fluorescent mobility markers. Anal. Chem. 2008, 80, 279-286. [CrossRef] [PubMed]

14. Dolník, V.; Liu, S.; Jovanovich, S. Capillary electrophoresis on microchip. Electrophoresis 2000, 21, 41-54. [CrossRef]

15. Guckenberger, D.J.; de Groot, T.E.; Wan, A.M.; Beebe, D.J.; Young, E.W. Micromilling: A method for ultra-rapid prototyping of plastic microfluidic devices. Lab Chip 2015, 15, 2364-2378. [CrossRef] 
16. Okagbare, P.I.; Emory, J.M.; Datta, P.; Goettert, J.; Soper, S.A. Fabrication of a cyclic olefin copolymer planar waveguide embedded in a multi-channel poly(methyl methacrylate) fluidic chip for evanescence excitation. Lab Chip 2010, 10, 66-73. [CrossRef]

17. Liu, K.; Xiang, J.Z.; Ai, Z.; Zhang, S.K.; Fang, Y.; Chen, T.; Zhou, Q.W.; Li, S.Z.; Wang, S.X.; Zhang, N.G. PMMA microfluidic chip fabrication using laser ablation and low temperature bonding with OCA film and LOCA. Microsyst. Technol. 2017, 23, 1937-1942. [CrossRef]

18. Chou, S.Y.; Krauss, P.R.; Renstrom, P.J. Imprint lithography with 25-nanometer resolution. Science 1996, 272, 85-87. [CrossRef]

19. Li, M.W.; Huynh, B.H.; Hulvey, M.K.; Lunte, S.M.; Martin, R.S. Design and characterization of poly(dimethylsiloxane)-based valves for interfacing continuous-flow sampling to microchip electrophoresis. Anal. Chem. 2006, 78, 1042-1051. [CrossRef]

20. McDonald, J.C.; Whitesides, G.M. Poly(dimethylsiloxane) as a material for fabricating microfluidic devices. Acc. Chem. Res. 2002, 35, 491-499. [CrossRef]

21. Jeong, Y.W.; Choi, K.W.; Kang, M.K.; Chun, K.J.; Chung, D.S. Transient isotachophoresis of highly saline samples using a microchip. Sens. Actuat. B Chem. 2005, 104, 269-275. [CrossRef]

22. Cui, H.; Dutta, P.; Ivory, C.F. Isotachophoresis of proteins in a networked microfluidic chip: Experiment and 2-D simulation. Electrophoresis 2007, 28, 1138-1145. [CrossRef]

23. Han, C.M.; Catoe, D.; Munro, S.A.; Khnouf, R.; Snyder, M.P.; Santiago, J.G.; Salit, M.L.; Cenik, C. Simultaneous RNA purification and size selection using on-chip isotachophoresis with an ionic spacer. Lab Chip 2019, 19, 2741-2749. [CrossRef] [PubMed]

24. Han, C.M.; Katilius, E.; Santiago, J.G. Increasing hybridization rate and sensitivity of DNA microarrays using isotachophoresis. Lab Chip 2014, 14, 2958-2967. [CrossRef]

25. Janasek, D.; Schilling, M.; Franzke, J.; Manz, A. Isotachophoresis in free-flow using a miniaturized device. Anal. Chem. 2006, 78, 3815-3819. [CrossRef] [PubMed]

26. Kim, J.; Kang, M.; Jensen, E.C.; Mathies, R.A. Lifting gate polydimethylsiloxane microvalves and pumps for microfluidic control. Anal. Chem. 2012, 84, 2067-2071. [CrossRef]

27. Kim, J.; Stockton, A.M.; Jensen, E.C.; Mathies, R.A. Pneumatically actuated microvalve circuits for programmable automation of chemical and biochemical analysis. Lab Chip 2016, 16, 812-819. [CrossRef]

28. Jang, L.W.; Lee, J.; Razu, M.E.; Jensen, E.C.; Kim, J. Fabrication of PDMS Nanocomposite Materials and Nanostructures for Biomedical Nanosystems. IEEE Trans. NanoBiosci. 2015, 14, 841-849. [CrossRef]

29. Khurana, T.K.; Santiago, J.G. Sample zone dynamics in peak mode isotachophoresis. Anal. Chem. 2008, 80, 6300-6307. [CrossRef]

30. Lim, A.E.; Lim, C.Y.; Lam, Y.C. Electroosmotic flow hysteresis for dissimilar ionic solutions. Biomicrofluidics 2015, 9, 024113. [CrossRef]

31. Routs, R.J. Electrolyte Systems in Isotachphoresis and Their Applications to Some Protein Seaparations; Solna Skriv- \& Stenograftjiinst AB: Solna, Sweden, 1971.

32. Jung, B.G.; Zhu, Y.G.; Santiago, J.G. Detection of $100 \mathrm{aM}$ fluorophores using a high-sensitivity on-chip CE system and transient isotachophoresis. Anal. Chem. 2007, 79, 345-349. [CrossRef] [PubMed]

33. Bahga, S.S.; Bercovici, M.; Santiago, J.G. Ionic strength effects on electrophoretic focusing and separations. Electrophoresis 2010, 31, 910-919. [CrossRef]

34. Krivankova, L.; Brezkova, M.; Gebauer, P.; Bocek, P. Importance of the counterion in optimization of a borate electrolyte system for analyses of anions in samples with complex matrices performed by capillary zone electrophoresis. Electrophoresis 2004, 25, 3406-3415. [CrossRef] [PubMed]

35. Garcia-Schwarz, G.; Rogacs, A.; Bahga, S.S.; Santiago, J.G. On-chip isotachophoresis for separation of ions and purification of nucleic acids. J. Vis. Exp. 2012, e3890. [CrossRef] [PubMed]

36. Hu, E.C.; He, J.G.; Liu, Z.H.; Ni, X.H.; Zheng, Y.G.; Gu, Q.; Zhao, Z.H.; Xiong, C.M. High levels of serum lactate dehydrogenase correlate with the severity and mortality of idiopathic pulmonary arterial hypertension. Exp. Ther. Med. 2015, 9, 2109-2113. [CrossRef] [PubMed]

37. Ma, L.J.; Gong, X.Y.; Yeung, E.S. Combinatorial screening of enzyme activity by using multiplexed capillary electrophoresis. Anal. Chem. 2000, 72, 3383-3387. [CrossRef]

38. Markert, C.L. Lactate dehydrogenase. Biochemistry and function of lactate dehydrogenase. Cell Biochem. Funct. 1984, 2, 131-134. [CrossRef] 
39. Chan, F.K.; Moriwaki, K.; De Rosa, M.J. Detection of necrosis by release of lactate dehydrogenase activity. Methods Mol. Biol. 2013, 979, 65-70. [CrossRef]

40. Chen, H.; Ahsan, S.S.; Santiago-Berrios, M.B.; Abruna, H.D.; Webb, W.W. Mechanisms of quenching of Alexa fluorophores by natural amino acids. J. Am. Chem. Soc. 2010, 132, 7244-7245. [CrossRef]

41. Cable, M.L.; Stockton, A.M.; Mora, M.F.; Willis, P.A. Low-temperature microchip nonaqueous capillary electrophoresis of aliphatic primary amines: Applications to Titan chemistry. Anal. Chem. 2013, 85, 1124-1131. [CrossRef]

42. Kim, J.; Jensen, E.C.; Stockton, A.M.; Mathies, R.A. Universal microfluidic automaton for autonomous sample processing: Application to the Mars Organic Analyzer. Anal. Chem. 2013, 85, 7682-7688. [CrossRef] [PubMed]

43. Jang, L.W.; Razu, M.E.; Jensen, E.C.; Jiao, H.; Kim, J. A fully automated microfluidic micellar electrokinetic chromatography analyzer for organic compound detection. Lab Chip 2016, 16, 3558-3564. [CrossRef] [PubMed]

44. Lee, J.; Estlack, Z.; Somaweera, H.; Wang, X.; Lacerda, C.M.R.; Kim, J. A microfluidic cardiac flow profile generator for studying the effect of shear stress on valvular endothelial cells. Lab Chip 2018, 18, 2946-2954. [CrossRef]

45. Linshiz, G.; Jensen, E.; Stawski, N.; Bi, C.; Elsbree, N.; Jiao, H.; Kim, J.; Mathies, R.; Keasling, J.D.; Hillson, N.J. End-to-end automated microfluidic platform for synthetic biology: From design to functional analysis. J. Biol. Eng. 2016, 10, 3. [CrossRef] [PubMed]

46. Kaigala, G.V.; Bercovici, M.; Behnam, M.; Elliott, D.; Santiago, J.G.; Backhouse, C.J. Miniaturized system for isotachophoresis assays. Lab Chip 2010, 10, 2242-2250. [CrossRef] [PubMed]

Publisher's Note: MDPI stays neutral with regard to jurisdictional claims in published maps and institutional affiliations. 